\title{
Influence of the Precursors and Chemical Composition of the Solution on the Properties of ZnO Thin Films Grown by Spray Pyrolysis
}

\begin{tabular}{|c|c|}
\hline Journal: & The Journal of Physical Chemistry \\
\hline Manuscript ID: & jp-2009-07990z.R2 \\
\hline Manuscript Type: & Article \\
\hline $\begin{array}{r}\text { Date Submitted by the } \\
\text { Author: }\end{array}$ & \\
\hline Complete List of Authors: & $\begin{array}{l}\text { Arca, Elisabetta; Trinity College Dublin, School of Physics } \\
\text { Fleischer, Karsten; Trinity College Dublin, School of Physics } \\
\text { Shvets, Igor; Trinity College Dublin, School of Physics; CRANN - } \\
\text { TCD }\end{array}$ \\
\hline
\end{tabular}

\section{S) ScholaroNE \\ Manuscript Central}


Influence of the precursors and chemical composition of the solution on the properties of $\mathrm{ZnO}$ thin films grown by spray pyrolysis

\author{
E. Arca ${ }^{1}$, K. Fleischer ${ }^{1 *}$, I. V.Shvets ${ }^{1}$ \\ ${ }^{1}$ Cleaner Energy Laboratory, School of Physics, Trinity College Dublin, Dublin 2, \\ Ireland
}

* Corresponding author: fleisck@tcd.ie 


\begin{abstract}
The effect of the solution composition on the properties of $\mathrm{ZnO}$ thin films grown by spray pyrolysis has been investigated. The $\mathrm{ZnO}$ films, both undoped and doped with either magnesium or aluminium, have been grown employing different solvents and different precursor salts. Afterwards the effects of these have been correlated to the morphology, optical and electrical properties of the films. In general it was found that organic salts are preferable over inorganic ones such as chlorides and nitrates. In the case of inorganic salts, unwanted etching processes, caused by acids formed as a result of the precursor decomposition lead to degradation of the films performance. Similarly, organic solvents are preferable over water due to a better droplet size distribution and, also, due to additional heat transfer towards the sample surface by their burning.
\end{abstract}

Keywords: doped zinc oxide, transparent conductive oxide, spray pyrolysis, resistivity, morphology $\mathrm{ZnO}: \mathrm{Al}$ 


\section{Introduction}

Spray pyrolysis is an inexpensive, versatile growth technique for thin films with a wide range of application spreading from solar cells technology (anti-reflective coatings, transparent conducting oxides (TCO) and absorber layers (CIS, CIS2) $)^{1-5}$ to gas sensors ${ }^{6}$, anodes for lithium-ion batteries ${ }^{7}$, optoelectronics devices ${ }^{5}$, as well as application in the glass industry ${ }^{8}$. Briefly, the spray-pyrolytic process consists of nebulizing a solution, containing the precursor salts, and transport towards a hot substrate by means of a gas stream $^{1,9,10}$. Within the hot zone above the substrate, the precursors undergo a pyrolytic decomposition, i.e. the thermal decomposition of the compound in the presence of oxygen ${ }^{11}$, and a thin layer is deposited onto the substrate.

Key parameters of this process are: the atomization technique, aerosol transport (carrier gas, pressure, distance and reactor geometry), substrate temperature and material, and most importantly, the chemical composition of the solution ${ }^{1,5}$.

Many efforts have been devoted to improve the performance of the nebulizing mechanism and different types of nozzles are commercially available today. ${ }^{1,12-15}$, . The crucial point is to get a homogeneous droplet size distribution as well as a small value for the mean particle size since better film quality is achievable in this case. ${ }^{1,16}$

The carrier gas determines the oxidizing potency of the atmosphere in which the pyrolytic reaction takes place ${ }^{5,17}$, hence influencing the cation oxidation number in the final product. Moreover, pressure in the nozzle determines the rate at which the droplets reach the surface, thus influencing both the growth rate and the cooling rate of the surface ${ }^{5,18}$. In case of the blast nozzle the pressure and gas type also determines the droplet size 
distribution. Hence it has a more direct influence in the final film properties.

The temperature of the substrate influences many different process parameters: aerosol transport towards the substrate, solvent evaporation, possibility of droplets impacting the surface and the dynamics of their spreading, and most importantly the precursor decomposition pathway ${ }^{1,19}$. Therefore, the surface temperature plays a major role in defining morphology and composition of the deposited film. In fact, by varying the temperature it is possible to change from regimes in which the precursor salts do not decompose to regimes associated with different oxidizing states possible for the precursor 20. The importance of the substrate material is mainly related to its heat capacity. Materials with a low heat capacity and conductivity are cooled much faster by the spray than those that have large ones. This influences the performance of the overall process and changes effective growth temperatures ${ }^{21,22}$. For all parameters discussed so far, there is a clear understanding of their role and, even if their refinement for each individual system has to be done experimentally, the trend and generic influence has been confirmed in many cases. Although some attempts have been made, ${ }^{23-25}$, the influence of the chemistry of the precursor solution has been less rigorously discussed. Only few general guidelines have been established. In particular it is known that solvents with lower density and surface tension (such as the alcohol-based ones) enable creating droplets of smaller size. Moreover solvents with lower boiling point vaporize easily and this can have a major consequence for achieving real pyrolytic decomposition ${ }^{15,18}$. Furthermore, a co-solvent can be added in order to tune the oxidizing potency of the atmosphere where the reaction takes place ${ }^{20}$. Highly soluble precursors are preferred, as well as volatile molecules are required as co-product of the pyrolytic decomposition ${ }^{9,23}$ 
In the present article, the main role of each chemical component of the solution has been studied for both solvents and salt precursors. Undoped as well as $\mathrm{Mg}$ and $\mathrm{Al}$ doped $\mathrm{ZnO}$ has been investigated as a prototype system, mainly due to the easy availability of different precursor salts and their high solubility in different solvents. For each salt a systematic variation of the temperature has been performed in order to find the most suitable growth temperature. Each precursor has been tested in a variety of solvents and, afterwards, a comparison between the different precursor solutions in otherwise identical conditions have been done. In particular, the role of both the solvent and the salts for the morphological, optical and electrical properties of the deposited films has been investigated.

\section{Experimental}

The spray deposition was carried out in a custom built confined environment (Abbess 18" Cube), employing an air blast atomizer nozzle (PNR Air assisted ultrasonic atomizer, model MAD $0331 \mathrm{~B} 1 \mathrm{BA}$ ) placed at a distance of $29 \mathrm{~cm}$ from the deposition substrate. Air was used as carrier gas and a mass flow controller (Vögtlin, model red-y) was used to regulate the flow. The oxygen concentration inside the chamber was monitored using an oxygen sensor (Sensortechnics, model XYA1) placed at a distance of $35 \mathrm{~cm}$ away from the nozzle. The substrate consists of glass slide (Fisher brand, thickness $0.8-1 \mathrm{~mm}$ ) kept at a constant temperature through a ceramic heater (Watlow model CER-1-01-00007). For the liquid delivery a peristaltic pump (Watson Marlow Pumps Peristaltic 520S) has been 
used.

In order to find out the most suitable decomposition temperature for each precursor, this was varied from 573 to $723 \mathrm{~K}$, with steps of 50 degrees. For the sake of simplicity the temperatures reported are the ones referred to the heater set point. However, in order to measure the thermal gradient across the glass slide and cooling power due to the air stream and the spray solvents, a type K thermocouple (nickel-chromium) was placed in test experiments within $1 \mathrm{~mm}$ above the glass surface, in a region where the reaction is expected to happen. For the actual sample growth this was removed to have an unobstructed sample surface.

For the synthesis, all the chemicals were purchased from Sigma-Aldrich and used without any further purification. The solvents employed were methanol, ethanol, both HPLC-grade, and deionised water. For selected samples, a mixture of them was used. For the $\mathrm{ZnO}$ synthesis, $\mathrm{Zn}\left(\mathrm{CH}_{3} \mathrm{CO}_{2}\right)_{2} \cdot 2 \mathrm{H}_{2} \mathrm{O}, \mathrm{Zn}\left(\mathrm{NO}_{3}\right)_{2} \cdot 6 \mathrm{H}_{2} \mathrm{O}$, and $\mathrm{ZnCl}_{2}$ were used as $\mathrm{Zn}$ source. In all cases a $0.2 \mathrm{M}$ solution was used.

The following precursor solutions have been used for sample growth.

Zinc acetate was employed both with water, methanol and a mixture of ethanol/methanol equal to $70 / 30$. The use of this has been necessary in order to speed up the dissolving process. Moreover in order to test the influence of water as solvent, mixtures with a ratio $\mathrm{CH}_{3} \mathrm{OH} / \mathrm{H}_{2} \mathrm{O}$ equal to $75 / 25,50 / 50,25 / 75$ have been tested as well.

The $\mathrm{ZnCl}_{2}$ precursor was employed either with water, methanol and ethanol, as well as in a mixture of $\mathrm{H}_{2} \mathrm{O} / \mathrm{CH}_{3} \mathrm{CH}_{2} \mathrm{OH}$.

The $\mathrm{Zn}\left(\mathrm{NO}_{3}\right)_{2} 6 \mathrm{H}_{2} \mathrm{O}$ precursor was used just in full methanol, based on the results for the other precursors. 
For all the synthesis the liquid flow rate was fixed to $2.66 \mathrm{ml} \mathrm{min}^{-1}$, the gas flow was $141 \mathrm{~min}^{-1}$. The best decomposition temperature was $623 \mathrm{~K}$ for the acetate and nitrate precursors while for the chloride the decomposition temperature was $673 \mathrm{~K}$.

Doping has been carried out employing $\mathrm{Mg}\left(\mathrm{CH}_{3} \mathrm{CO}_{2}\right)_{2} \cdot 4 \mathrm{H}_{2} \mathrm{O}$ and $\mathrm{MgCl}_{2} 6 \mathrm{H}_{2} \mathrm{O}$ as magnesium source, $\mathrm{AlCl}_{3} \cdot 9 \mathrm{H}_{2} \mathrm{O}$ and $\mathrm{Al}\left(\mathrm{NO}_{3}\right)_{3} \cdot 9 \mathrm{H}_{2} \mathrm{O}$ and $\mathrm{AlOCH}\left(\mathrm{CH}_{3}\right)_{23}$ for aluminium. For the dissolution of the aluminium isopropoxide, a mixture of $70 \%$ isopropanol and $30 \%$ methanol has been used. The nominal molar concentration ratio of these doping agents was $[\mathrm{Mg}] /[\mathrm{Zn}]=10 \%$ and $[\mathrm{Al}] /[\mathrm{Zn}]=4 \%$. These values have been chosen taking into consideration the data reported in literature ${ }^{26-34}$. In order to find the optimum concentration, regarding film quality and conductance, we screened a larger range of $\mathrm{Al} / \mathrm{Zn}$ and $\mathrm{Mg} / \mathrm{Zn}$ ratios. For the scope of this paper we hence focus on the already optimized values. Due to the small fraction of doping agent present, all other parameters were kept as reported above for the undoped $\mathrm{ZnO}$.

The crystallographic analysis was made by X-ray diffraction (XRD) employing a Bruker D8 Discovery (Bragg Brentano configuration, Cu-tube, Göbel mirror, $0.2 \mathrm{~mm}$ horizontal entry slit, $4.5 \mathrm{~mm}$ soller entry slit, $2.5 \mathrm{~mm}$ soller exit slit, horizontal exit slit via a LynxEye stripe detector $(0.075 \mathrm{~mm}))$. The resulting XRD patterns were analyzed by carrying out a numerical reconstruction of line profiles according to the Rietveld method. For this purpose, the MAUD software was employed

The morphological analysis was carried out employing an atomic force microscope , AFM, (NT-MDT SPM Solver PRO NT-MDT, tip NSC35/AIBS) or the optical microscope (Leitz Laborlux $12 \mathrm{ME}$ with standard bright field and interference contrast capabilities, fitted with a Leica DFC420 Camera, PC controlled), depending on the 
roughness of the sample.

The optical properties (transmission) were determined by a Cary 50 UV-Vis-NIRspectrophotometer equipped with a Xenon lamp.

The electrical measurements have been carried out with conventional four point probe method or a simple 2 wire method for low conducting samples (Agilent E3634A constant current source at 2-5mA, Agilent 34401A multimeter) using gold capped spring loaded contacts in a linear arrangement with pitch of $7.5 \mathrm{~mm})$.

\section{Role of solvent}

The role of solvent has been tested employing both zinc acetate and zinc chloride as precursors. Using the organic precursor, the $\mathrm{ZnO}$ layer was obtained employing water, methanol or a mixture of them. Several films have been grown changing the solvent composition (ratio between water and methanol) as following: 100/0, 75/25, 50/50, 25/75, 0/100. All the other parameters were kept fixed. Despite the different solvent composition, from the crystallographic point of view, all the samples consist of just one phase, zincite (PDF number 01-074-0534), with hexagonal structure (Fig. 1). No traces of unreacted precursors have been found (acetate: PDF-033-1464, chloride: PDF-01-0721285). The refinement carried out with MAUD indicates that the cell parameters are: $a$ =3.246, $c=5.258 \AA$. The sample grown in pure methanol and the one with a solvent composition containing $25 \%$ of water show dominantly (002) oriented crystals, while for all the others, a good fit can be obtained only assuming that the sample is composed of different micro-crystallites, some of which have a (002) and others have (100) preferred 
orientation.

The relationship between the morphology of the sample and the solvent composition is straightforward. Smooth, good quality layer can be obtained just by using methanol as solvent while the surface roughness gets with increasing water content (Fig. 2). The morphology of the film has a direct influence on the optical properties of the coating (Fig. 3). Increasing water content leads to a decrease in the transparency of the film. It is worth noting that also the growth rate increases with the water content. This has already been observed in literature in the case of the $\mathrm{SnO}_{2}{ }^{35}$. Nevertheless the above mentioned decrease in transmission is not caused by the thickness of the sample, but it is a consequence of the scattering losses at the rough surface. Only the methanol grown film is smooth enough to observe typical Fabry-Perot oscillations of thin transparent films. In order to verify this, samples with a comparable thickness have been grown employing a mixture of 50/50\% water/methanol and $100 \%$ methanol. For this purpose, the growth time has been shortened to account for the higher growth rate of the water-based solution in comparison with the pure methanol one. For these layers of similar thickness the ones grown with water are always rougher and hence show a lower transmission. Optical band gap values as determined by linear extrapolation of the transmission data are found to be unaffected by the choice of solvent and was found to be $3.35 \pm 0.05 \mathrm{eV}$.

In order to understand the major cause for the roughness, some basic parameters have been monitored during the growth. As stated before, the droplet size and the temperature of the surface play a crucial role for the growth of a good layer. With respect to the particle size, it is easy to understand that solvent with a low density create droplets with lower size, which don't impinge onto the surface. In this case the vaporization of the 
solvent happens just above the surface leading to a heterogeneous reaction ${ }^{35}$. Therefore, it can be argued that, for this particular type of nozzle, the lower density solvent creates droplets with smaller size and more homogeneous distribution, improving the performance of the process. Nevertheless, this is not the only role played by the solvent. In order to confirm this, three samples were grown using pure water, pure methanol and a mixture ethanol/methanol (70/30). For each sample the oxygen concentration in the pyrolysis chamber has been monitored during the growth. The relative decrease in the oxygen concentration suggests that the solvent burns during the process (Fig. 4). In fact the oxygen concentration drops down for all of the three solvents used but, in the case of the organic solvent the decrease is much larger. In the water case the decrease is due to the change in chemical composition of the atmosphere inside the chamber as water vapour replaces air, while for the organic solvent the change is either due to the combustion products (mainly water and $\mathrm{CO}_{2}$, even if the presence of the co-product cannot be excluded), due to solvent that doesn't burn and due to the oxygen consumption caused by the burning of the solvent. As the solvent burns mostly in the vicinity of the surface, the exothermic burning will provide additional heating to the salt decomposition. In order to confirm this hypothesis, the heater power supplied needed to keep its temperature constant, has been analyzed. To keep the heater at $423 \mathrm{~K}$ during the deposition in the case of water, $63 \mathrm{~W}$ was needed, while in the case of methanol less power has been delivered $(50 \mathrm{~W})$. As the temperature within the heater is a function of cooling power of the sprayed solution and the thermal conductivity of the substrate, these results already show the importance of an active temperature control, as the reduction in surface temperature is partly compensated by the adjustment in heater power, leading to 
more reproducible surface temperatures. However, even with this active compensation, different solvent have shown different cooling rates which influence, to different extent, the actual surface temperature. To quantify these, a thermocouple has been attached to the glass slide surface and the heater has been set to a constant power value able to keep it at a similar temperature. In this way the effect of the closed loop system has been cancelled. Due to its position, the temperature monitored is relative to the layer of air just above the glass surface (within $1 \mathrm{~mm}$ ), where the reaction is supposed to happen. The cooling power due to the air stream itself, the water spray and due to two organic solvents has been measured. The data revealed that the temperature above the glass slide is $651 \mathrm{~K}$ when the heater reading is $615 \mathrm{~K}$. Due to the cooling caused by the air stream, the glass slide temperature drops down to $591 \mathrm{~K}$. When the water is sprayed, the surface temperature decreases further to $573 \mathrm{~K}$, while for methanol the temperature doesn't vary with respect the value measured for the air stream. In this case no precursor salt has been added to the solvent and therefore the properties measured are related just to the solvent used and hence universally applicable to any other pyrolysis growth process. It can be seen that the largest drop of surface temperature of $\sim 60 \mathrm{~K}$ is caused by the air stream. Absolute values of this decrease are highly dependent on the heat capacity of the heater, and also the air pressure. For piezo-electric nebulizers this drop should be less significant due to the lower gas flow.

Water has shown a greater cooling rate due to its evaporation (18 degrees), while the organic solvent burns, hence compensating the cooling. In fact considering the enthalpy evaporation process for both the solvent $\left(\Delta_{\mathrm{ev}} \mathrm{H}\left(\mathrm{H}_{2} \mathrm{O}\right)=40 \mathrm{~kJ} / \mathrm{mol}, \Delta_{\mathrm{ev}} \mathrm{H}\left(\mathrm{CH}_{3} \mathrm{OH}\right)=35\right.$ $\mathrm{kJ} / \mathrm{mol}$ ) and the respective sprayed moles ( 0.15 for water and 0.067 for methanol), the 
drop in temperature for methanol should be roughly half of the water one. In other words, assuming a drop in temperature of 18 degrees for water, the expected value for methanol should be 8 degrees. The fact that no temperature drop was observed can be explained if we accept that the methanol burns in the process thus releasing energy. If the heater temperature is controlled by a closed loop system, the drop caused by the air blow or water sprayed is lower compared to the open loop $(50 \mathrm{~K}$ vs $60 \mathrm{~K}$ for air and 15 vs 18 for water). The reason is that the heat loss due to air/water is partially compensated by increased power of the heater. Within the error of our measurement no difference in either oxygen concentration or surface temperature has been found between methanol and ethanol. This can be explained only if the burning of the ethanol is incomplete. In fact the standard enthalpy of combustion of ethanol and the relative oxygen consumption are higher than for methanol. Nevertheless, the calculation of the overall quantity of oxygen inside the chamber shows that this is not enough to completely burn the solvent sprayed.

Although direct temperature measurements have been performed only with pure solvents, our indirect measurements (required heater power and oxygen consumption), carried out during deposition, show that these effects are generically applicable for solutions including different precursor salts. In fact, when the same tests have been repeated for the chloride precursor, higher power consumption and lower oxygen depletion have been observed for water than for the organic solvent. Again, no significant differences in heater power and oxygen concentration between methanol and ethanol solutions have been observed. The general nature of this behavior can be explained as the low quantity of salt present in the solution $(\sim 0.2 \mathrm{M})$ does not significantly alter the solvent burning process. However, absolute values may differ due to different spray rates, 
temperature or even initial conditions of the growth atmosphere.

\section{Role of the precursor salt}

As stated before, smooth, good quality layer can be obtain by using zinc acetate in methanol. In contrast using zinc chloride led to rough discontinued layers, either in combination with organic solvents, water or a mixture of them. We relate this to different co-products that are formed during the pyrolytic decomposition of the two different precursors.

If zinc acetate is used, the pyrolytic decomposition leads to the formation of zinc oxide according to the following reaction:

$$
\mathrm{Zn}\left(\mathrm{CH}_{3} \mathrm{CO}_{2}\right)_{2} \cdot 2 \mathrm{H}_{2} \mathrm{O} \stackrel{\Delta}{\longrightarrow} \mathrm{ZnO}+\mathrm{CH}_{3} \mathrm{COCH}_{3}, \mathrm{CH}_{3} \mathrm{CO}_{2} \mathrm{H}, \mathrm{CO}_{2}
$$

The thermal decomposition of this precursor has been studied in literature using the thermal gravimetric analysis (TG) coupled to the differential thermal analysis (DTA) ${ }^{36}$. The results show that the organic chain undergoes a series of reactions that ends up with the production of volatile molecule, such as acetone, acetic acid and carbon dioxide), while the zinc ions react with the oxygen present in the atmosphere or in the water to form the correspondent oxide. This is deposited as a thin film onto the glass slide. The chloride decomposition instead, leads to the formation of $\mathrm{HCl}$ as co-product according to the following reaction:

$$
\mathrm{ZnCl}_{2}+\mathrm{H}_{2} \mathrm{O} \stackrel{\Delta}{\longrightarrow} \mathrm{ZnO}+2 \mathrm{HCl}
$$

The poor surface morphology of the films grown using $\mathrm{ZnCl}_{2}$ can thus be attributed to the presence of $\mathrm{HCl}$, which induced a chemical etching of the surface, leading to a rough, 
not closed film (Fig 5). This explanation is supported by the detection of the zinc chloride hydroxide phase, $(\mathrm{ZnClOH}, \mathrm{PDF}$ n 01-072-0525, Fig. 6) in the sample grown employing a mixture of ethanol-water solution at $623 \mathrm{~K}$. According to the TG-DTA studies reported in literature ${ }^{37}$, the decomposition of this oxy-chloride is a complex process that involves different pathways and intermediates, that ends with the formation of the hydrochloric acid and zinc oxide. Therefore it can be concluded that the decomposition of the precursor actually occurs, but, the intimate contact between the acid and the oxide leads to the degradation of the latter one.

The same reason can be advocated to explain the roughness of the film obtained by using zinc nitrate as precursor (PDF-25-1231 ,Fig. 7). In this case the overall reaction is:

$$
\mathrm{Zn}\left(\mathrm{NO}_{3}\right)_{2} \cdot 6 \mathrm{H}_{2} \mathrm{O} \stackrel{\Delta}{\longrightarrow} \mathrm{ZnO}+\mathrm{NO}_{2}+\mathrm{NO}+\mathrm{O}_{2}+6 \mathrm{H}_{2} \mathrm{O}
$$

Nevertheless, the TG-DTA studies reported in literature ${ }^{38,39}$, established that the thermal decomposition of the $\mathrm{Zn}\left(\mathrm{NO}_{3}\right)_{2} \cdot 6 \mathrm{H}_{2} \mathrm{O}$ is again a complex process, for which different pathways are available. In particular the decomposition of the nitrate fraction can occur directly with the formation of the nitrogen oxides species $\left(\mathrm{NO}_{2}\right.$ and $\left.\mathrm{NO}\right)$ or can involve the formation of many different hydroxide species as intermediates, leading to the formation of nitric acid as intermediate and its further decomposition. According to other authors, the decomposition pathway of these species is temperature dependent, and for intermediate values, the formation of $\mathrm{HNO}_{3}$ has been confirmed. At higher temperatures, the direct decomposition represents the main reaction. So, even if the temperature for the decomposition of this compound has been set in the latter range, it is worth noting that while the precursors are carried towards the substrate, they experience a thermal gradient which may lead to the formation of the corresponding acid. Thus, it can be argued that 
the same basic explanation carried out to justify the chemical behavior of the chloride precursor, can be extended also to the nitrate one. In other words, the formation of a strong acid as intermediate or final product of the pyrolytic reaction leads to the degradation of the film which thus results in a high roughness. We have shown here, that for $\mathrm{ZnO}$ growth this etching process is limiting the quality of pyrolysis grown films. Although the acid formation is a generic problem when chlorides and nitrates are used, the actual impact for a given oxide can be quite different as it depends on the stability of the oxide with respect to $\mathrm{HCl}$ and $\mathrm{HNO}_{3}$. The etching process can have a significant impact not only to the morphology but also the growth rate itself and even texturing, if there are different etching rates for different crystallographic faces.

In order to confirm the general nature of the problem of acid formation for the $\mathrm{ZnO}$ growth, a doping study of the films has been done employing different precursors as the cation source. If this is a generic problem, poorer films are expected whenever inorganic salts were used as doping agent. In fact, in the case of significant Mg doping (10\%), no closed film can be obtain if chloride salts have been used as zinc and magnesium source, while closed films have been obtained if zinc acetate was used. In this case, doping has been made both with chloride and acetate as magnesium source. Even the relatively small amount of $\mathrm{MgCl}_{2}$ already leads to a degradation of film quality and a higher transparency is obtained for the organic precursor (Fig. 8). The rougher sample due to the etching process from $\mathrm{HCl}$ formed with the $\mathrm{MgCl}_{2}$ precursor has a lower $\mathrm{UV}$ transmission due to the enhanced scattering losses. Introducing $\mathrm{Mg}$ into the $\mathrm{ZnO}$ lattice does affect the optical band gap, which was found to be $3.42 \pm 0.05 \mathrm{eV}$.

The same approach has been adopted for the Al doping. In this case the nitrate, the 
chloride and an organic precursor have been tested. As in the previous case, when the chloride or nitrate precursors were used, the film was rough and less transparent. If the organic precursor was used, smooth and highly transparent films were obtained (Fig. 9). In this case significant variation in the optical band gap determined by the transmission measurements occurred. The values if the aluminium isopropoxide is used is $3.36 \pm 0.05$ $\mathrm{eV}$, while for the nitrate and chloride $3.4 \pm 0.1 \mathrm{eV}$ and $3.45 \pm 0.1 \mathrm{eV}$ are measured. However it is not clear whether these changes are systematic errors in the determination for the rough, cracked films, differences in the actual aluminium incorporation or an actual influence of the precursor on the band structure of the material. Further investigations are required to clarify that finding.

\section{Influence of the precursor on electrical properties}

We have shown so far that the choice of solvent influences the morphology and optical properties of the film through the droplet size distribution, and also change in the surface temperature due to the solvent burning. Secondly we have shown that acid formation from inorganic salts in the reactive atmosphere increases surface roughness and hence decreases transmission. As aluminium-doped $\mathrm{ZnO}$ is used as transparent conducting oxide, we also wanted to test the influence of the Al-precursor salt on the electrical properties.

All the $\mathrm{ZnO}$ :Al films have been grown in methanol with zinc acetate as zinc precursor. They showed a sheet resistance $\left(R_{s h}\right)$, in the $\mathrm{M} \Omega$ region. Films using aluminium chloride and aluminium isopropoxide showed sheet resistances of 1.5 and $1 \mathrm{M} \Omega / \square$ respectively, 
films with aluminium nitrate showed a sheet resistance of $21.5 \mathrm{M} \Omega / \square$. As the growth rates and hence film thickness varied, the resistivity $(\rho)$ of the film has been calculated by normalizing the sheet resistance with respect to the measured thickness of the sample $(t)$, according to the following relationship:

$$
R_{s h}=\rho / t
$$

The thickness value has been determined by a least square fit of the measured transmittance, using an optical multilayer model and published optical constants for $\mathrm{ZnO}$ 40, and simply by the slide weight. The values determined optically for the sample obtained with the organic, nitrate and chloride precursors are 340, 185 and $165 \mathrm{~nm}$, respectively, while the values determined through the weight are: 300, 280 and $230 \mathrm{~nm}$. As it can be seen, a good agreement is settled between the two estimations in the case of the organic precursor, while the optical evaluation underestimated the thickness of the sample for the two inorganic precursors. This has to be attributed again to the roughness of the latter two samples that tends to dampen the Fabry-Perot oscillation. Considering the optical thickness, the resistivities are: $34 \Omega \mathrm{cm}^{-1}, 400 \Omega \mathrm{cm}^{-1}$ and $25 \Omega \mathrm{cm}^{-1}$ respectively for the isopropoxide, nitrate and chloride aluminium precursor. So it can be seen that the chloride and organic precursor lead to a resistivity in the same order of magnitude. In case of the chloride, the value is underestimated due to the systematic error in the optical thickness evaluation of the rough sample. The nitrate is significantly worse by one order of magnitude. Obviously the choice of precursor influences not only the morphology of the film, and thus its optical properties, but also the electrical ones. In order to determine potential differences in the conduction mechanism, temperature dependent measurements have been performed in a range from $50-100^{\circ} \mathrm{C}$. The 
conductivity is proportional to the number of carriers $(n)$ and their mobility $(\mu)$ according to the following relationship ${ }^{41}$ :

$$
\sigma=q\left(\mu_{n} n_{d}+\mu_{h} n_{a}\right)
$$

Where $\mu_{n}$ and $\mu_{h}$ are the electron and hole mobilities and $n_{d}$ and $n_{a}$ the electron (donor) and hole (acceptor) numbers. For the dominantly n-type $\mathrm{ZnO}$ films presented here, however, the contribution of the minority carrier to the conductance can be neglected. With a nominal $\mathrm{Al}$ content of $4 \%$ it is reasonable to assume that not all donors are thermalised at room temperature. The increase in conductance is then dominated by the increase in thermally excited carriers following an exponential dependence:

$$
n_{d} \sim \exp \left(-E_{a} / k_{b} T\right)
$$

It is worth noting that $E_{a}$ is related to the donor binding energy through the following expression: $E_{a}=1 / 2\left(E_{c}-E_{d}\right)$, provided that $E_{c}$ is the acceptor level of the conduction band and $E_{d}$ the donor level. Hence from a linear fit in an Arrhenius plot of the conductance vs. temperature measurements (Figure 10), the activation energy $E_{a}$ and hence the donor binding energy can be extracted, assuming that there is a negligible temperature effect on the mobility itself. The activation energies determined are $16 \mathrm{meV}$ for the organic precursor, $33 \mathrm{meV}$ for the nitrate precursor and $20 \mathrm{meV}$ for the chloride one. The latter once are in agreement with previously reported donor binding energies $\left(E_{c}-E_{d}\right)$ of the $\mathrm{Al}$ donor of $53-65 \mathrm{meV}^{42,43}$. The better conductance of the film grown with the organic 
precursors is supposed to originate from the significantly lower donor binding energy. Further studies are required to confirm this explanation and to identify the donor. It is likely that the presence of reactive hydrogen or possibly carbon leads to the formation of $\mathrm{Al}-\mathrm{H}, \mathrm{Al}-\mathrm{C}, \mathrm{Zn}-\mathrm{H}, \mathrm{Zn}-\mathrm{C}, \mathrm{O}-\mathrm{H}$ or O-C defect complexes, leading to a different donor level $^{42,43}$.

Unfortunately the determination of activation energies via resistance measurements alone is not enough to verify this hypothesis as there is the possibility that despite the high doping levels all carriers are already thermalised and the exponential dependence of the conductance with temperature arises from a grain boundary scattering limited transport. In this case the dependence of the carrier concentration with the temperature is negligible, while the mobility of the carriers varies with temperature as ${ }^{44}$ :

$$
\mu=\mu_{0}(T)^{1 / 2} \exp \left(-E_{b} / k_{B} T\right)
$$

$E_{b}$ represents the thermal activation energy of the scattering process through the grain boundary. Assuming that changes in the term $\mu_{0}(T)^{1 / 2}$ are small in comparison with the exponential term over the temperature range measured (323 to $373 \mathrm{~K}$ ), the linear fit Arrhenius plot (Figure 10) would provide an estimate of this barrier. It is possible that since the films grown with aluminium isopropoxide are significantly smoother than those grown with aluminium chloride or nitrate, the microscopic structure of the grain boundaries varies. In addition less grain boundaries and hence a higher conductance are expected, however the slope of the curve should only change if also the microstructure of the boundaries is significantly changed. 
In both cases the higher resistance for the nitrate precursor (in comparison to the chloride) can be directly correlated to a reduced number of carriers. Further analyses are required to identify the reason for that, but one of the hypotheses can be the actual incorporation of the oxygen created by the decomposition of the nitrate moiety inside the grain boundaries.

To conclude, the discussion reported so far shows that the choice of precursor salt for the dopant, influences not only the morphology as described above but also the conductance. In fact, the effective number of incorporated dopants might change with the type of salt, as well as the type of donor, possibly due to the formation of defect complexes including other elements in the precursor such as hydrogen and carbon in the case of organic precursors. Alternatively the microstructure of grain boundaries is modified. With the present data both models or a combination of them are possible, and direct measurements of carrier concentration and mobility over a wider temperature range (in particular at low temperatures), as well as quantitative measurements of the aluminium content would be required to distinguish them.

\section{Conclusion}

A systematic study on the influences of different solvents and precursor salts on the performance of the spray pyrolytic process of $\mathrm{ZnO}$ and $\mathrm{ZnO}: \mathrm{Al}$ has been presented. In particular the role of the solvent has been correlated to the optical properties of the layers deposited. A correlation between the physical and chemical properties of the solvent, the type of nozzle used and the quality of the layer has been established. Summarizing, when 
relative simple air blast nozzles are used, a low density, volatile solvent is required in order to get smaller droplets size and favour the evaporation of the solvent itself. Moreover, the burning of the organic solvent during the deposition has been demonstrated. It has been established that this process can actually compensate the cooling power of the liquid in itself, affecting one of the most important parameters of the entire process: the actual temperature at which the reaction occurs.

The role of the precursor salt has been systematically investigated with three different types of precursor, namely the nitrate, the chloride and an organic one (isopropoxide for $\mathrm{Al}$, acetate for $\mathrm{Mg}$ and $\mathrm{Zn}$ ). For all the three cations, the best results have been achieved employing the organic salt. The reason for this can be ascribed to the decomposition of the organic moiety which leads to the formation of volatile, inert products. Instead, the anion moiety decomposition can lead to the formation of strong acids as intermediate or final products, and higher film roughness have been observed. This has been attributed to the chemical etching that these acid species induce. The roughness so created, determines a degradation of the optical properties of the compound. Secondly, the choice of doping precursor salt can influence the type of carrier responsible for the electrical properties (via the formation of different defect complexes), their concentration (due to differences precursor decomposition rate) and possibly variations in the grain boundary microstructure (different overall morphology).

\section{Acknowledgment}

The authors would like to acknowledge financial support from Enterprise Ireland under grant PC/2007/0367. 


\section{References}

(1) Perednis, D.; Gauckler, L.J. J. Electroceramics 2005, 14, 103-111

(2) Nanu, M.; Schoonman, J.; Goossens, A. Nano Letters 2005, 5, 1716-1719

(3) Goossens, A.; Hofhuis, J. Nanotechnolgy 2008, 19, 424018

(4) Isac, L. et al. Thin Solid Films 2007, 515, 5755- 5758

(5) Blandenet, G.; Courts, M.; Lagarde, Y. Thin Solid Films 1981, 77, 81-90

(6) Korotcenkov, G. et al. J. Phys. Conf. Ser. 2005, 15, 256-261

(7) Ng, S. H. et al. J. Phys. Chem. C 2007, 111, 11131-1113

(8) Mochel, J. M. U.S. Patent: 2,564,707, 1951

(9) Roger, C. et al. Nanostructured Materials 1994, 4, 529-535

(10) Sears, W. M.; Gee, M. A. Thin Solid Films 1988, 165, 265-2736

(11) Schwartz, R. W.; Schneller, T.; Waser, R. Comptes Rendus Chimie 2004, 7, 433-461

(12) Eslamian, M.; Ashgriz, N. J. Eng. Mat. Technol. 2007, 129, 130-143

(13) Heine, M.C.; Pratsinis, S. E. Ind. Eng. Chem. Res. 2005, 44, 6222- 6232

(14) Neagu, R; Perednis, D.; Princivalle, A.; Djurado E. Chem. Mater. 2005, 17, 902-910

(15) Patil, P. S.; Mat. Chem. Phys. 1999, 59, 185-198

(16) Wang, M. et al. J. Phys. Chem. C 2008, 112, 1920-1924

(17) Ocampo, E.; Arce, R.; Koropecki, R. R; Buitrago, R. H. Sol. Energy Mater. Sol. Cells 1995, 36 , 327-337

(18) Wang, W.N. et al. Ind. Eng. Chem. Res. 2008, 47, 1650- 1659 
(19) Bouzidi, A. et al. Mat. Sci. Eng. B 2003, 97, 5-8

(20) Kim, J. H. et al. J. Mater. Res. 2003, 18, 1614-1622

(21) Muecke, U. P.; Messing, G. L.; Gauckler, L. J. Thin Solid Films 2009, 517, 15151521

(22) Muecke, U. P. et al. Thin Solid Films, 2009, 517, 1522-1529

(23) Chen, C.Y. et al. Ceram. Int. 2008, 34, 409-416

(24) Hernández-Fenollosa, M.A. et al., Thin Solid Films 2008, 516, 1622-1625

(25) Bacaksiz, E. et al. J. Alloys Comp. 2008, 466, 447-450

(26) Zhang, X. et al. Thin Solid Films 2005, 429, 248-252

(27) Zhang, X. et al Appl. Phys. Lett. 2005, 87, 092101

(28) Oshawa, T. et al Chem. Mat. 2009, 21, 144-150

(29) Yoshino, K.; Oyama, S.; Yoneta, M. J. Mater. Sci 2008, 19, 203-209

(30) De Merchant, J.; Cocivera, M. Chem. Mater. 1995, 7, 1742-1749

(31) Lee, J.H.; Park, B. O. Mat. Sci. Eng. B 2004, 106, 242-245

(32) Mohammad, M. T.; Hashim, A.A.; Al-Maamory, M. H. Mat. Chem. Phys. 2006, 99, $382-387$

(33) Seeber, W.T. et al Mat. Sci. Semicon. Proc. 1999, 2, 45-55

(34) Hu, J.; Gordon, R. G. J. Appl. Phys. 1992, 71, 880-890

(35) Vasu, V.; Subrahmanyam, A. Thin Solid Films 1990, 193-194, 973-980

(36) Lin, C. C.; Li, Y. Y. Mat. Chem. Phys. 2009, 113, 334-337 
(37) Garzia-Martinez, O. et al J. Mat. Sci. 1994, 29, 5429-5434

(38) Maneva, M.; Petrov, N. J. Therm. Anal. 1989, 35, 2297-2303

(39) Biswick, T. et al. J. Solid State Chem. 2007, 180, 1171-1179

(40) Rebien, M.; Henrion, W.; Bär, M.; Fischer, Ch.-H. Appl. Phys. Lett. 2002, 80, 35183520

(41) Yu, P. Y.; M. Cardona, Fundamentals of Semiconductors, Physics and Materials

Properties, $2^{\text {nd }}$ edition; Springer: Berlin, 1999; p 195

(42) Meyer, B.K. et al. Semicond. Sci. Technol 2005, 20, S62-S66

(43) Look, D.C. Mat. Sci. Eng. B 2001, 80, 383-387

(44) Chopra, K. L.; Major, S.; Pandya, K. Thin Solid Films 1983, 102, 1-46 
Figure 1: XRD patterns of $\mathrm{ZnO}$ samples grown with zinc acetate in varying solvent composition. The corresponding composition water/methanol ratios are A: 100/0, B: 75/25, C: 50/50, D: 25/75, E: 0/100. The main peaks relative to the zincite phase are indicated.

Figure 2: Morphology of the samples grown with different solvent composition. Images A-D have been taken with an optical microscope, while $\mathrm{E}$ is an AFM picture. The bar in the optical images stands for $500 \mu \mathrm{m}$. The corresponding composition water/methanol ratios are A: 100/0, B: 75/25, C: 50/50, D: 25/75. Sample E grown in pure methanol had no visible contrast in the optical microscope and an AFM image is shown instead. It is clear that size and number of large circular crater caused by the impact of the largest unevaporated droplets are significantly reduced with increasing methanol content.

Figure 3: Transmission measurements of samples grown with varying solvent composition. From the bottom to the top, the ratio water/methanol is $100 / 0,75 / 25,50 / 50$, 25/75, 0/100.

Figure 4: Oxygen concentrations as a function of the growth time for water (dotted), methanol (solid) and a mixture ethanol/methanol (70/30, dashed) as solvent. The more significant drop in the case of organic solvents is caused by the oxygen consumption due to burning. 
Figure 5: Morphology of the $\mathrm{ZnO}$ samples grown employing zinc chloride as precursor. The red bar equals $500 \mu \mathrm{m}$.

Figure 6: XRD patterns of the sample grown employing a mixture ethanol/water (70/30) as solvent and zinc chloride as precursor. The peaks are relative to the phase $\mathrm{ZnClOH}$.

Figure 7 Optical microscope imagine referred to the $\mathrm{ZnO}$ sample grown employing zinc nitrate as precursor. The bar equals $500 \mu \mathrm{m}$

Figure 8: Transmission measurements of $\mathrm{ZnO}: \mathrm{Mg}$ samples. The solid curve refers to the sample where magnesium acetate was used, while the dotted one is the sample where magnesium chloride was used.

Figure 9: Transmission measurement of $\mathrm{ZnO}$ :Al samples grown employing aluminium isopropoxide (dotted curve), aluminium nitrate (dashed one) and aluminium chloride (solid one) as source

Figure 10: Conductance data as a function of the inverse of temperature. Samples have been grown with different aluminium sources: (-) aluminium isopropoxide, (8) aluminium chloride, and $(\forall)$ aluminium nitrate. The slopes (least square fits shown) give the thermal activation energy for the main carrier of 34, 61, $66 \mathrm{meV}$ respectively. 
1

2

3

4

5

6

7

8

9

10

11

12

13

14

15

16

17

18

19

20

21

22

23

24

25

26

27

28

29

30

31

32

33

34

35

36

37

38

39

40

41

42

43

44

45

46

47

48

49

50

51

52

53

54

55

56

57

58

59

60

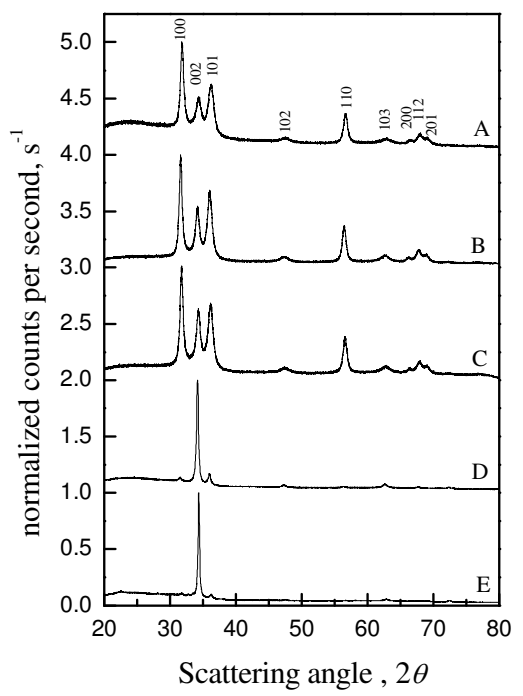

Fig 1 

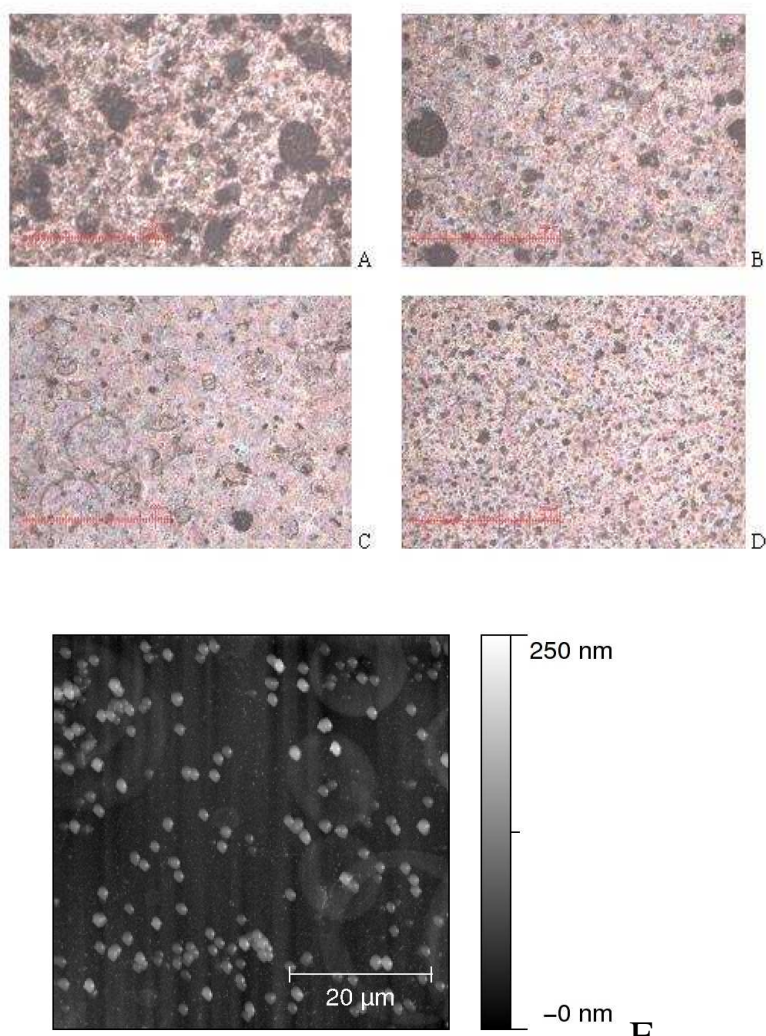

$250 \mathrm{~nm}$

Fig 2 
1

2

3

4

5

6

7

8

9

10

11

12

13

14

15

16

17

18

19

20

21

22

23

24

25

26

27

28

29

30

31

32

33

34

35

36

37

38

39

40

41

42

43

44

45

46

47

48

49

50

51

52

53

54

55

56

57

58

59

60

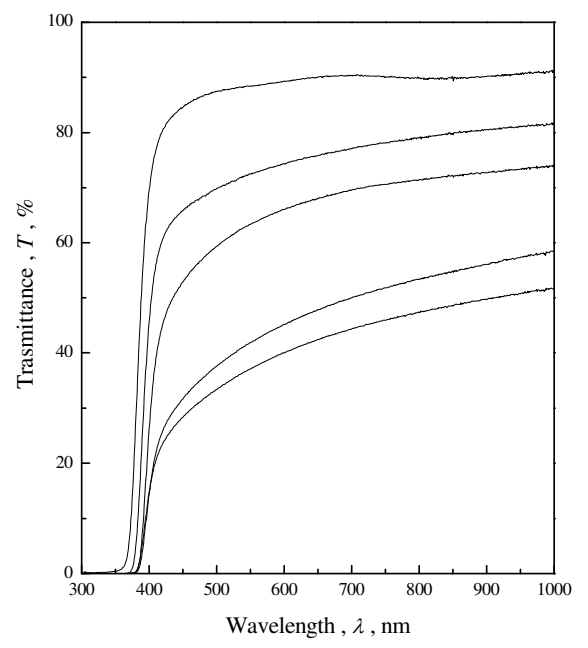

Fig 3 


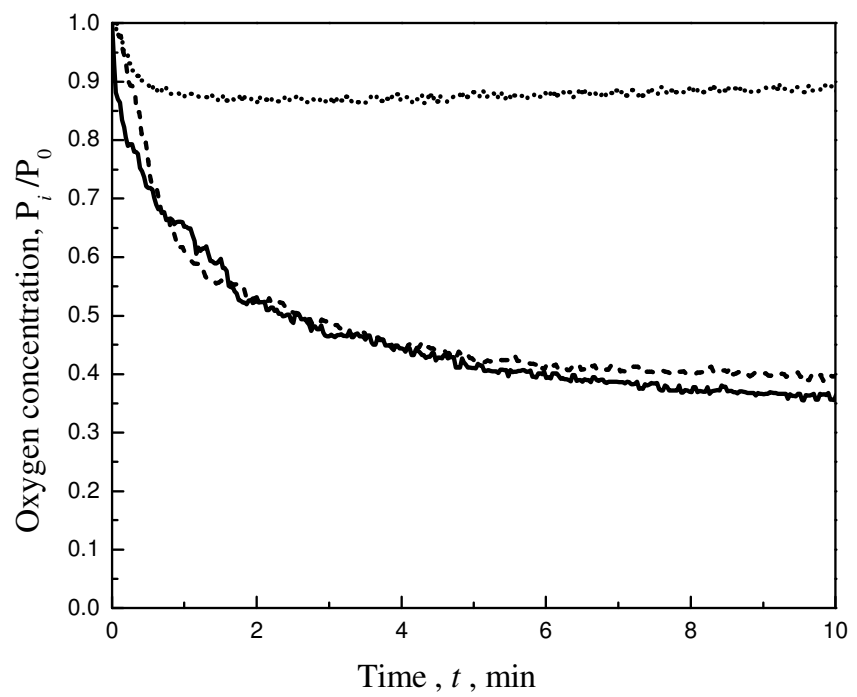

Fig 4 


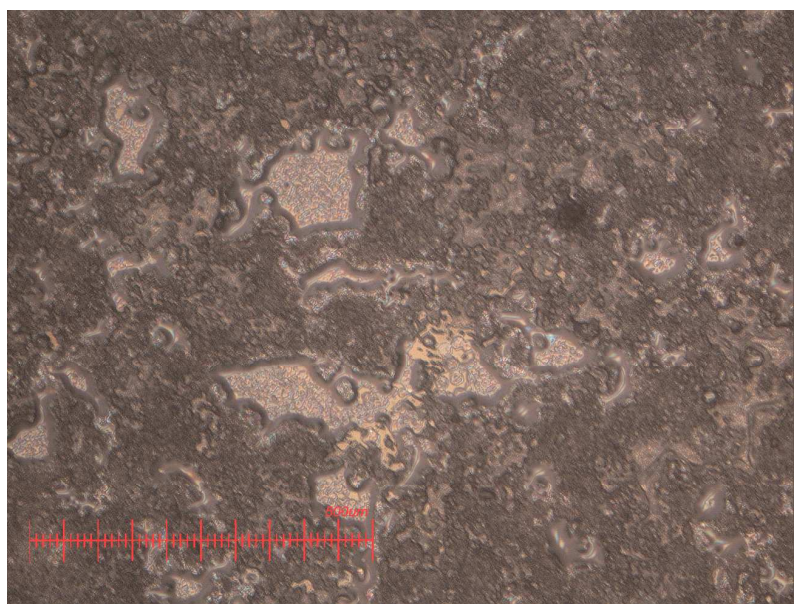

Fig 5 


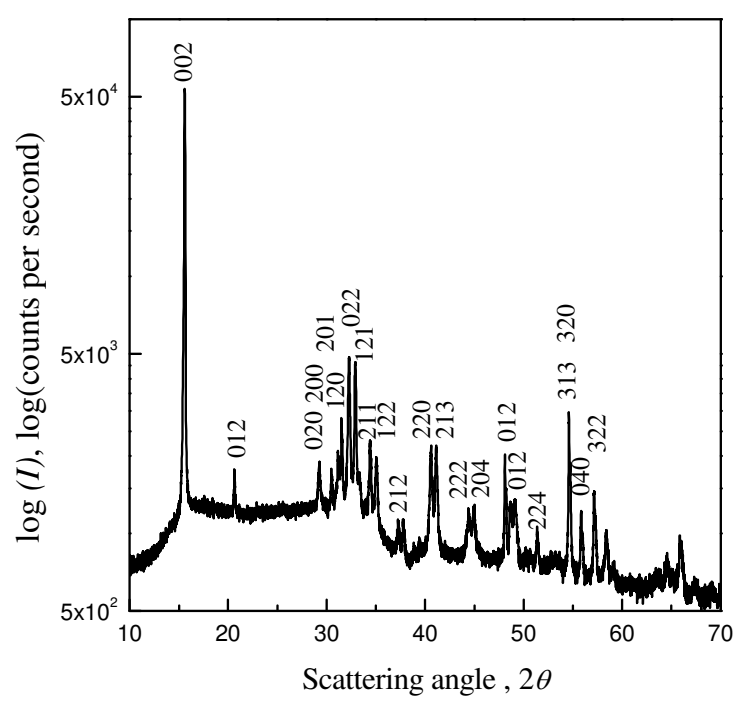

Fig 6 


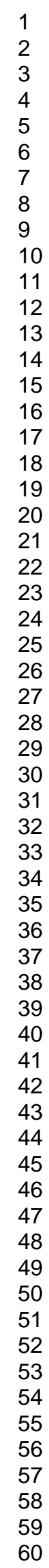

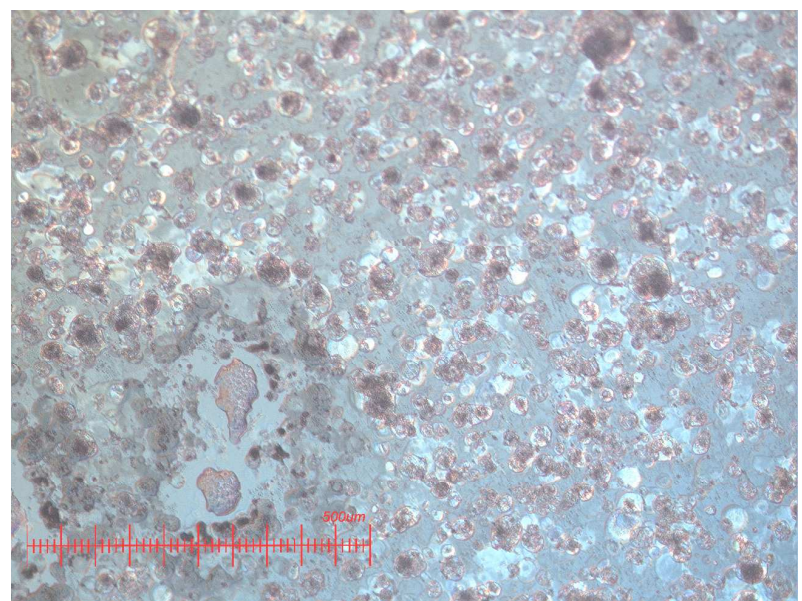

Fig 7 


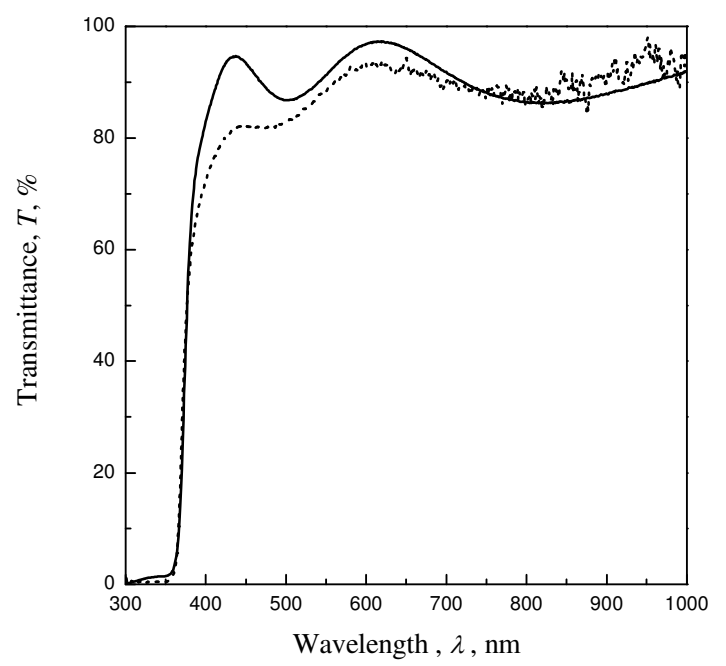

Fig 8 
1

2

3

4

5

6

7

8

9

10

11

12

13

14

15

16

17

18

19

20

21

22

23

24

25

26

27

28

29

30

31

32

33

34

35

36

37

38

39

40

41

42

43

44

45

46

47

48

49

50

51

52

53

54

55

56

57

58

59

60

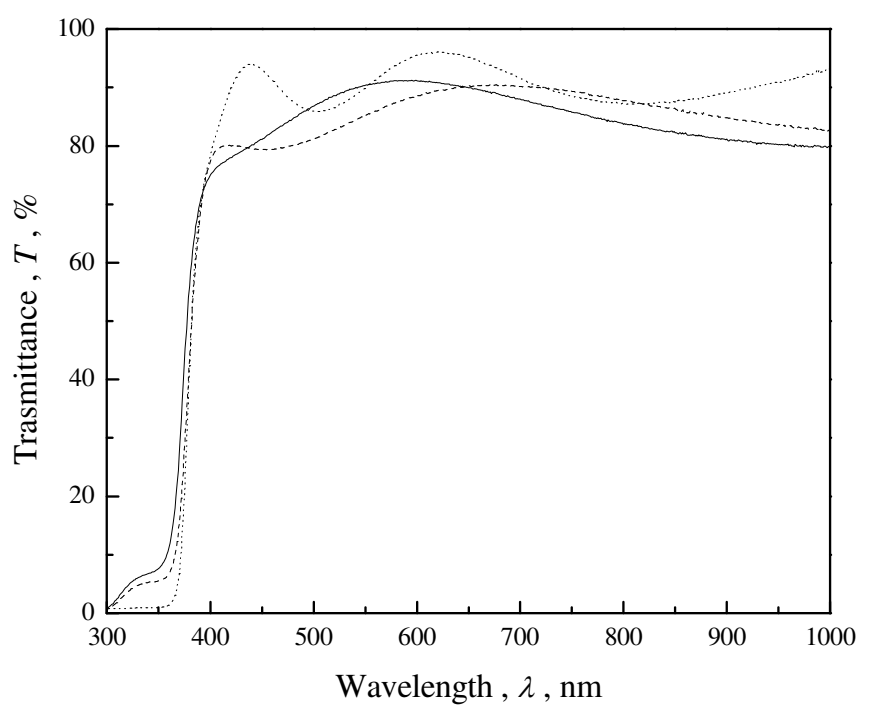

Fig 9 


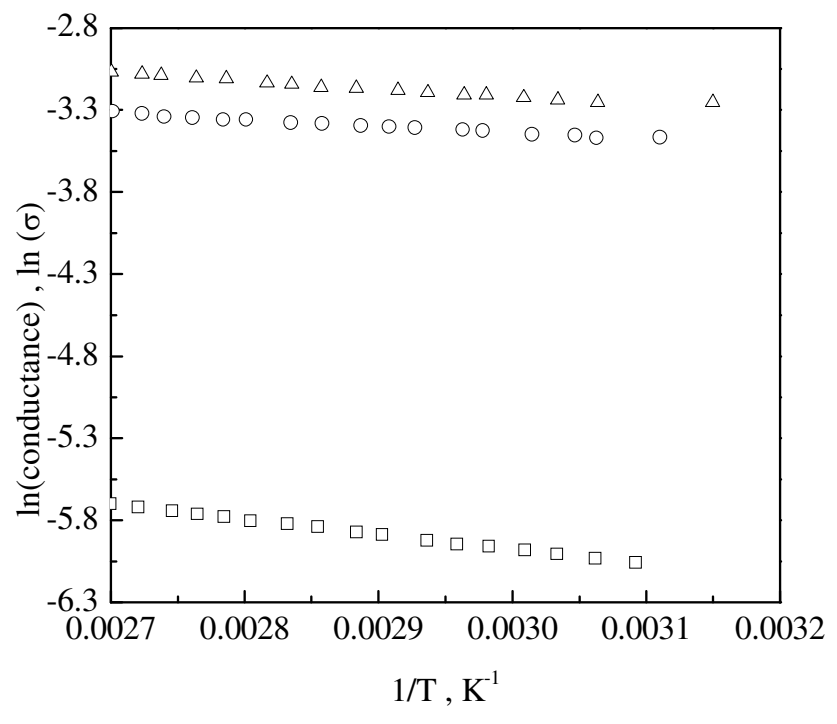

Fig 10 


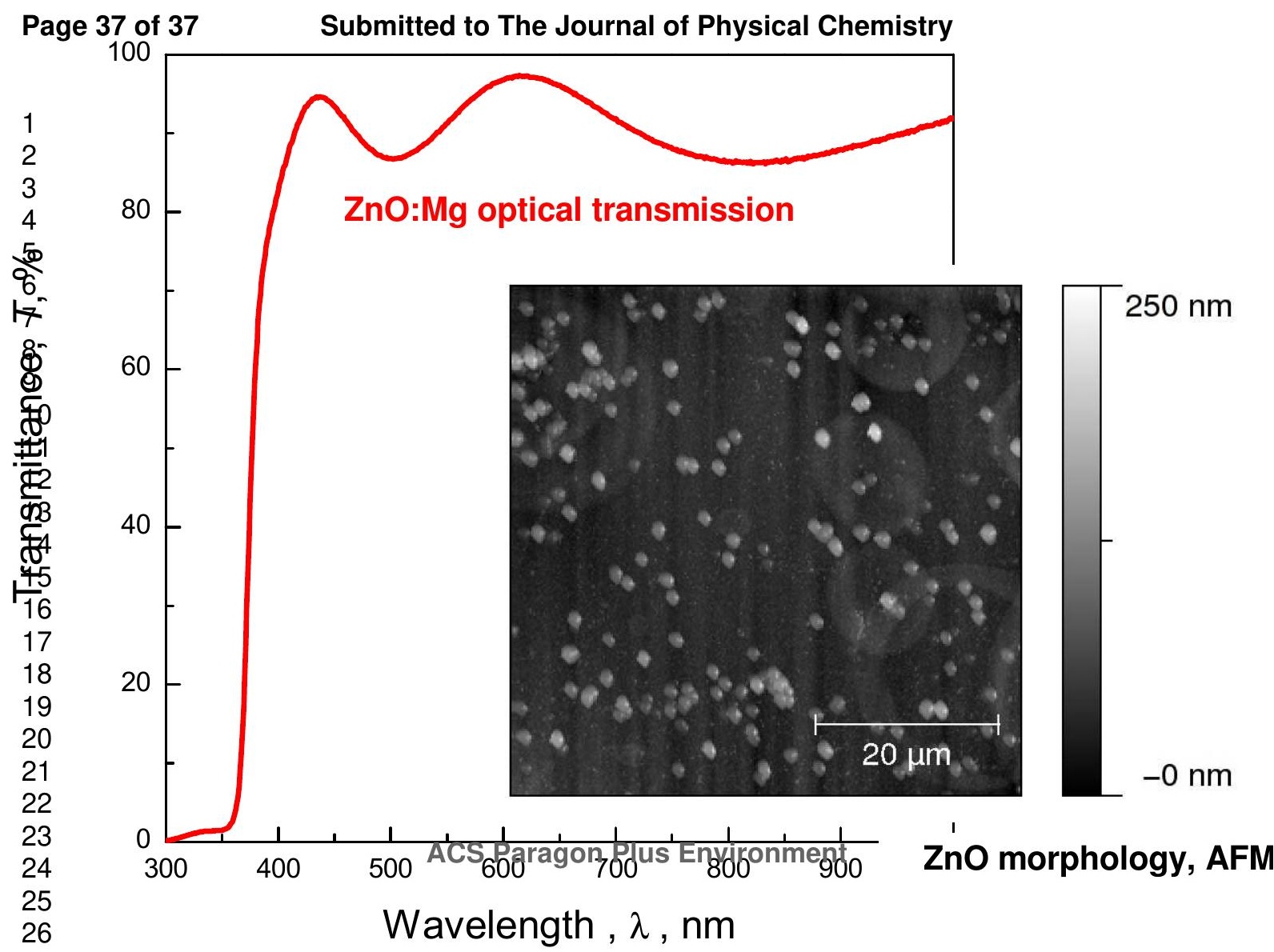

